\title{
Heat sink design considerations in medium power electronic applications with long power cycles
}

\author{
Panagiotis Asimakopoulos, Konstantinos Papastergiou \\ CERN, Geneva, Switzerland, \\ Torbjörn Thiringer, Massimo Bongiorno \\ Chalmers University of Technology, Gothenburg, Sweden
}

Keywords: «Thermal design», «Cooling», «DC-DC converter», «Power cycling», «Physics research».

\begin{abstract}
The aim of this work is to investigate the impact of the heat sink thickness and material, as well as, of the convection coefficient of the water cooling system on the power-electronics module thermal stressing. The heat extraction capability of different thicknesses is tested. It is concluded that the thickest heat sink results in marginally lower temperature variation at the junction level compared to the second thickest one. In the thickest heat sink case, the linear dependence of the thermal resistance on the thickness counteracts the benefit of the increased thermal capacitance. The increase in the cooling medium flow rate, which corresponds to an increase in the convection coefficient between the heat sink bottom surface and the water, can be avoided by increasing the thickness of the heat sink. In this way, the energy consumption of the cooling system is reduced. The increase in the flow rate drastically reduces the thermal stressing in the thinnest heat sink case. The increase of the heat sink thickness can be beneficial for long power cycles. Copper and aluminum are the two materials that are compared, with the copper heat sink exhibiting a slightly increased performance. The study is conducted for a medium power DC-DC converter of a magnet supply with focus on long power cycles.
\end{abstract}

Presented at: EPE 2015, 7-10 September 2015, Geneva, Switzerland

Geneva, Switzerland

October, 2015 


\title{
Heat sink design considerations in medium power electronic applications with long power cycles
}

\author{
Panagiotis Asimakopoulos, \\ Chalmers University of Technology \\ CERN, European Laboratory for Particle Physics \\ Konstantinos Papastergiou \\ CERN, European Laboratory for Particle Physics \\ CH-1211, 23, \\ Geneva, Switzerland \\ E-Mail: panagiotis.asimakopoulos@cern.ch \\ E-Mail: k.papastergiou@cern.ch
}

\author{
Torbjörn Thiringer, \\ Massimo Bongiorno \\ Chalmers University of Technology \\ Hörsalvägen 11, \\ Gothenburg, Sweden \\ E-Mail: torbjorn.thiringer@,chalmers.se \\ E-Mail: massimo.bongiorno@chalmers.se
}

\section{Keywords}

«Thermal design», «Cooling», «DC-DC converter», «Power cycling», «Physics research».

\begin{abstract}
The aim of this work is to investigate the impact of the heat sink thickness and material, as well as, of the convection coefficient of the water cooling system on the power-electronics module thermal stressing. The heat extraction capability of different thicknesses is tested. It is concluded that the thickest heat sink results in marginally lower temperature variation at the junction level compared to the second thickest one. In the thickest heat sink case, the linear dependence of the thermal resistance on the thickness counteracts the benefit of the increased thermal capacitance. The increase in the cooling medium flow rate, which corresponds to an increase in the convection coefficient between the heat sink bottom surface and the water, can be avoided by increasing the thickness of the heat sink. In this way, the energy consumption of the cooling system is reduced. The increase in the flow rate drastically reduces the thermal stressing in the thinnest heat sink case. The increase of the heat sink thickness can be beneficial for long power cycles. Copper and aluminum are the two materials that are compared, with the copper heat sink exhibiting a slightly increased performance. The study is conducted for a medium power DC-DC converter of a magnet supply with focus on long power cycles.
\end{abstract}

\section{Introduction}

The thermal aspect in the design of a power electronic system is one of the determining factors for a reliable, prolonged and efficient operation of the system. The power electronic modules are subject to high thermal stress that can negatively impact the lifetime of the components. Critical thermal-stressing parameters often found in the literature are, in significance order, the junction temperature swing, the maximum junction temperature and the pulse length. According to [1], the number of cycles to failure of a power module can be expressed as a function of the dominant thermal-stressing parameters

$$
N_{f}=A\left(\Delta T_{j}\right)^{b_{1}} e^{\left(\frac{Q}{R T}\right)} t_{o n}^{b_{2}}
$$

where $\Delta T_{j}$ is the junction temperature swing, $T_{j m}$ the maximum junction temperature, $t_{o n}$ the pulse length, $A, b_{1}, b_{2}$ are constants obtained experimentally that characterize the module, $R$ is the universal gas constant and $Q$ is the activation energy. More parameters can be added if the module structure is known in detail [1]. 
Assuming that the Silicon chip temperature is kept below the upper operation limit of $150^{\circ} \mathrm{C}$ or $175^{\circ} \mathrm{C}$, the most vulnerable parts [2], in a standard power device structure [3], are the bond wires, the solder layer between the chip and the substrate and the solder layer between the substrate and the baseplate. Manufacturers often provide estimations of the power module lifetime referring to the junction temperature and not to the other vulnerable parts, since most of the failure modes are directly connected to the chip.

The purpose of this paper is to investigate different aspects of the heat sink and cooling system design aiming to reduce the thermal stress for a power module exposed to a long pulse due to its application. More in detail, a target is to investigate different materials and thickness values for the heat sink [4] via simulation using PLECS [5] by recording the junction and heat sink temperature. Furthermore, by running an $\mathrm{AC}$ sweep, the Bode magnitude plot of the output heat flow is obtained and is another indication of the cooling system effectiveness. A final goal is to test two different convection coefficients for the cooling system. Finally, a lifetime estimation is discussed. The power modules are not placed in a high-radiation area, therefore the radiation failure mechanism is not taken into consideration in this work.

\section{Description of the application}

The application of interest is the power conversion in particle accelerators for nuclear physics research. The example of an electromagnet power supply is employed. The main structure of the magnet supply system consists of an AC-to-DC converter linking the system with the grid, a DC link and a DC-to-DC converter (H-bridge) controlling the magnet current, see Fig. 1.

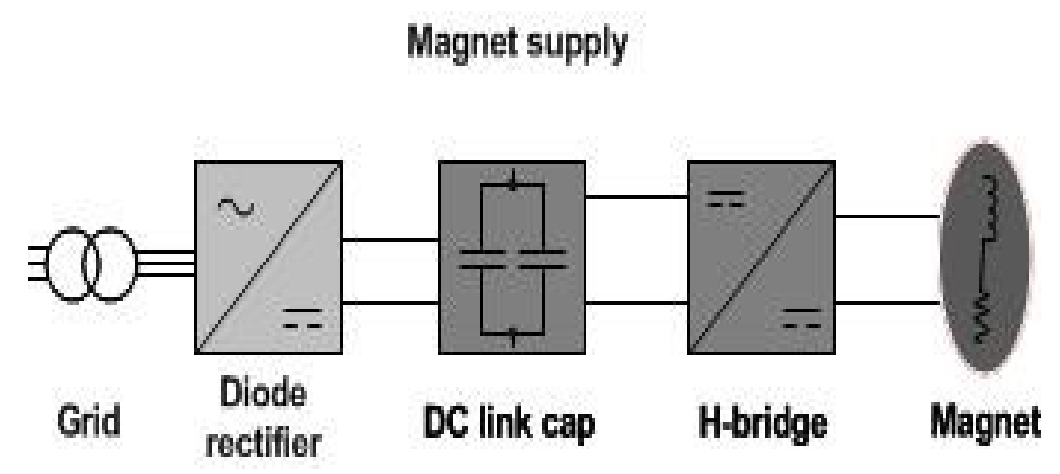

Fig. 1: Magnet supply system

The focus in this work is on the H-bridge. The DC link nominal voltage is $900 \mathrm{~V}$ and the current shape provided to the magnet is considered to be a trapezoidal pulse with a magnitude of $600 \mathrm{~A}$, a ramp-up and ramp-down time of $1 \mathrm{~s}$ each and a total on-time of $8 \mathrm{~s}$ within a time period of $100 \mathrm{~s}$. This is one of the cycles that a converter could be expected to operate at, in reality with a shorter turn-off time to combine it with other cycles within the $100 \mathrm{~s}$. If the converter is designed for a single load current profile, it can be designed to reduce the impact of a specific current profile, by examining various aspects for instance by investigating the impact of the heat sink thickness. A similar load profile could, also, occur in traction or wind turbine applications. This specific mission profile of the semiconductor module might then cause a high thermal stress in terms of junction temperature swing and a decreased number of maximum amount of cycles.

\section{Method}

\section{Simulation models}

For the considered H-bridge, four single-switch, ABB IGBT power modules 5SNA 1600N170100 [6], each having an antiparallel diode with a current rating of $1600 \mathrm{~A}$ and a voltage rating of $1700 \mathrm{~V}$, are used. The selection of the power modules electrical ratings seems to be conservative but the focus in this application is on the long lifetime. Therefore the thermal performance of the module is, also, crucial for 
the design. The thermal model of the module is created using the PLECS thermal library and the total magnet supply system is simulated. Only the simulation of the H-bridge is electro-thermal, in order to calculate the power losses for each semiconductor device.

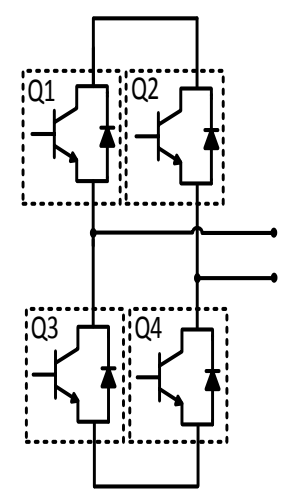

(a)

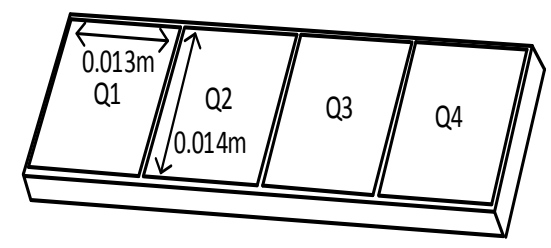

(b)

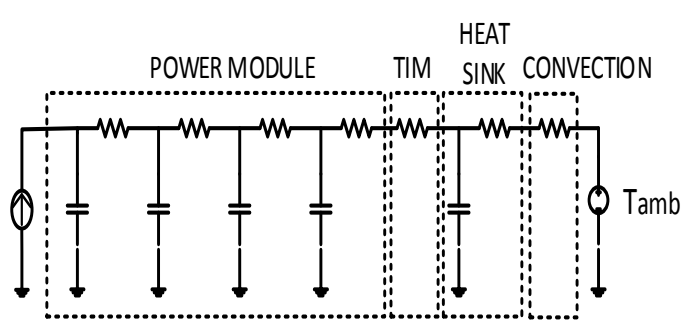

(c)

Fig. 2: (a) H-bridge electronic circuit, (b) mechanical layout, (c) thermal model of IGBT/diode junction-to-ambient

Each IGBT and each diode thermal network is fed with its power dissipation profile. All networks are connected in series with a common thermal network that represents the Thermal Interface Material (TIM), heat sink and heat convection to the ambient. Conclusively, the considered thermal coupling among different devices is at the level of the TIM. From this model, one can vary the heat sink and heat convection parameters and, directly observe the impact at the junction and heat sink temperature.

Moreover, a second model is built to perform an AC sweep analysis for the heat produced at the mostly stressed device of the H-bridge. . Due to the inductive load DC profile and based on the simulation results, the mostly stressed devices are the upper left and lower right IGBTs having an equal load, followed by the upper right and lower left antiparallel diodes This set-up can be represented by a Cauer network [7] consisting of the models of the upper right IGBT, the TIM, the heat sink and the convection to the cooling medium. In this way the Bode plot for the heat removed to the ambient in relation to the heat input at the device is obtained, covering the range of frequencies included in the application. This diagram is an indication of the ability of the system to extract the heat to the ambience.

\section{Heat sink design}

At this stage, the thermal impedance of the TIM, the heat sink and the interface between the heat sink and the ambient are calculated, in order to build the complete Cauer network from junction to cooling medium. The TIM is a regular silicon paste.

The heat sink area is selected to be equal to the total area of the four power modules, equal to four times the single module area $(0.130 \mathrm{~m} \times 0.140 \mathrm{~m}$ for the selected modules, see Fig. 2). It is regarded that the heat is spread uniformly in the baseplate and, then, into the heat sink. The geometry of the heat sink is in this work chosen to be a simple orthogonal brick. The thickness of the heat sink at the module side is varied, see Fig. 3 to observe its impact on the thermal stressing of the module.

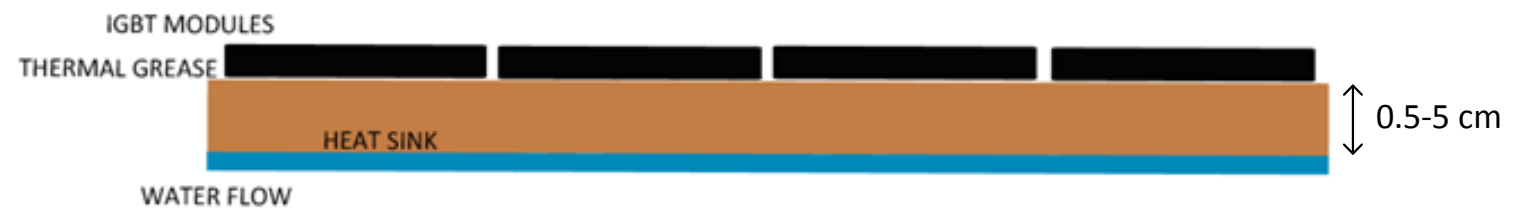

Fig. 3: Power stack structure 
The materials under investigation for the heat sink are aluminum and copper. Aluminum exhibits high specific weight heat capacity and low density. On the other hand, its thermal conductivity is lower than that of copper. Table I demonstrates the relevant material characteristics of aluminum and copper.

Table I: Aluminum and copper thermal properties of interest

\begin{tabular}{|c|c|c|}
\hline & Aluminum & Copper \\
\hline Density $\left[\mathrm{kg} / \mathrm{m}^{3}\right]$ & 2700 & 8960 \\
\hline $\begin{array}{c}\text { Thermal conductivity } \\
{\left[\mathrm{W} / \mathrm{m}^{\circ} \mathrm{C}\right]}\end{array}$ & 238 & 400 \\
\hline $\begin{array}{c}\text { Specific heat capacity } \\
{\left[\mathrm{J} / \mathrm{kg}{ }^{\circ} \mathrm{C}\right]}\end{array}$ & 900 & 385 \\
\hline $\begin{array}{c}\text { Specific heat capacity } \\
{\left[\mathrm{J} / \mathrm{dm}^{3}{ }^{\circ} \mathrm{C}\right]}\end{array}$ & 2430 & 3449.6 \\
\hline
\end{tabular}

The thermal capacitance and resistance [8] of the heat sink are calculated for the different thickness values.

$$
\begin{gathered}
R_{t h, h s}=\frac{d_{h s}}{\lambda_{h s} A_{h s}} \\
C_{t h, h s}=c_{h s} \rho_{h s} d_{h s} A_{h s}
\end{gathered}
$$

where $\rho_{h s}$ is the material density, $\lambda_{h s}$ the thermal conductivity, $A_{h s}$ the heat sink surface area and $c_{h s}$ the specific heat capacity. It is observed that for the same dimensions, the thermal resistance and the thermal capacitance ratios of aluminum over copper are approximately

$$
\begin{aligned}
& R_{t h, A l / C u}=1.68 \\
& C_{t h, A l / C u}=0.70
\end{aligned}
$$

The thermal resistance of the aluminum heat sink is substantially higher than that for the copper heat sink. The opposite is valid for the thermal capacity. For long cycle applications, the use of a copper heat sink could be beneficial due to its high specific heat capacity. For stationary systems as the magnet power supply of this application, the weight disadvantage of copper is not important. If the surface of the heat sink is increased, the copper heat spreading angle advantage, due to its higher thermal conductivity, could be exploited, in order to avoid increasing the thermal resistance and, possibly, the weight. A simulation of the heat flow with a Finite Element Method (FEM) [9] software tool could be more accurate, but it is out of the scope of this work. The heat sink thickness values selected are relatively high, in order to compensate for the thermal capacitance that is lost due to the limited surface area of the heat sink. This choice has a negative impact on the thermal resistance of the heat sink.

Table II shows the different thickness values and the corresponding thermal resistance and capacitance values of the aluminum and the copper heat sink body. As a first observation, copper, as expected, exhibits better thermal performance than aluminum. Copper price is higher than aluminum [10], therefore the decrease of the copper heat sink dimensions, due to its high thermal performance, could make this solution attractive. 
Table II: Thermal characteristics of aluminum and copper heat sink for different values of thickness

\begin{tabular}{|c|c|c|c|c|}
\hline \multirow{2}{*}{ Thickness $[\mathrm{cm}]$} & \multicolumn{2}{|c|}{ Thermal capacitance $\left[\mathrm{J} /{ }^{\circ} \mathrm{C}\right]$} & \multicolumn{2}{c|}{ Thermal resistance $\left[{ }^{\circ} \mathrm{C} / \mathrm{W}\right]$} \\
\hline & Aluminum & Copper & Aluminum & Copper \\
\hline 0.5 & 881 & 1220 & $2.88 * 10^{-4}$ & $1.38 * 10^{-4}$ \\
\hline 1 & 1760 & 2440 & $5.77 * 10^{-4}$ & $2.76 * 10^{-4}$ \\
\hline 3 & 5280 & 7320 & $17 * 10^{-4}$ & $8.28 * 10^{-4}$ \\
\hline 5 & 8810 & 12200 & $28.8 * 10^{-4}$ & $14 * 10^{-4}$ \\
\hline
\end{tabular}

The next step is to define the heat sink convection coefficient to the ambience [11], [12]. The heat is assumed to be extracted only from the bottom side of the heat sink. A moderate water-cooling convection coefficient, $5 \mathrm{~kW} /{ }^{\circ} \mathrm{C}^{*} \mathrm{~m}^{2}[13]$ is used in this work. The thermal resistance from heat sink-toambient is calculated with the expression

$$
R_{t h, h s-a}=\frac{1}{h A_{h s}}
$$

where $h$ is the convection coefficient and $A_{h s}$ the heat sink surface area .

\section{Results}

Using the thermal model of the H-bridge, the temperature is recorded throughout the power cycle at the top of the heat sink and at the junction of the upper left IGBT switch. For all figures the focus is on the first fifteen seconds of the cycle, where the most interesting part of the response takes place. For every case, within the $100 \mathrm{~s}$ cycle, the junction temperature returns to the initial value that is very close to the water inlet temperature of $25^{\circ} \mathrm{C}$.

Figures $4 \mathrm{a}$ and $4 \mathrm{~b}$ illustrate the junction temperature for the different values of thickness between 0.5 $\mathrm{cm}$ and $5 \mathrm{~cm}$ for the aluminum and the copper heat sink. The heat convection coefficient in both cases is $5 \mathrm{~kW} /{ }^{\circ} \mathrm{C}^{*} \mathrm{~m} 2$. The temperature response is almost identical for all considered thickness values at the first second. According to [6], this time period represents the time constant of the module, therefore for pulses shorter than one second, the heat sink makes no difference. For the $0.5 \mathrm{~cm}$ heat sink, the thermal capacitance is very low leading to a high maximum temperature of approximately $85^{\circ} \mathrm{C}$ and, also, to a high temperature swing from the maximum temperature to the ambient temperature. Similar observations are valid for the $1 \mathrm{~cm}$ heat sink, with a maximum temperature of $78{ }^{\circ} \mathrm{C}$.

For the two thick heat sinks the difference is small, although the increase in the thickness is significant. The $3 \mathrm{~cm}$ heat sink limits the junction temperature to a maximum of $68{ }^{\circ} \mathrm{C}$ and the temperature swing to $43.5^{\circ} \mathrm{C}$. In the $5 \mathrm{~cm}$ heat sink case, the increased thermal resistance, due to the increased thickness, obstructs the heat extraction and the thermal capacitance is not high enough to filter the temperature swing. For the $5 \mathrm{~cm}$ heat sink the temperature swing is $40.1{ }^{\circ} \mathrm{C}$ and the maximum temperature around $65^{\circ} \mathrm{C}$. The $3 \mathrm{~cm}$ heat sink is selected for the aluminum case, due to its dimension and weight advantage over the $5 \mathrm{~cm}$.

In the copper heat sink case, see Fig. $4 \mathrm{~b}$, the observations are the same as in the aluminum case. Despite the better thermal characteristics of copper, its increased cost outweighs the benefit in the temperature variation. If the $3 \mathrm{~cm}$ heat sink is compared for aluminum and copper, the lead of copper is only $2^{\circ} \mathrm{C}$ for the maximum temperature and the swing. 
(a)

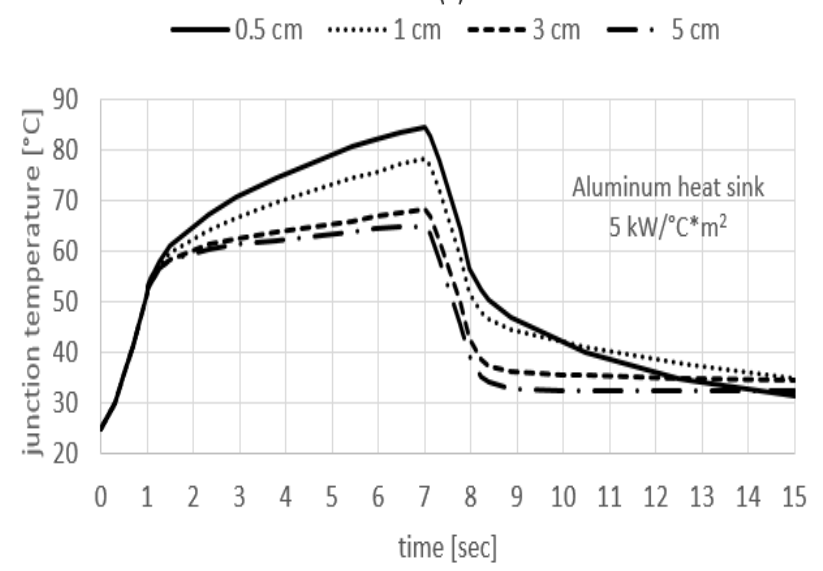

(b)

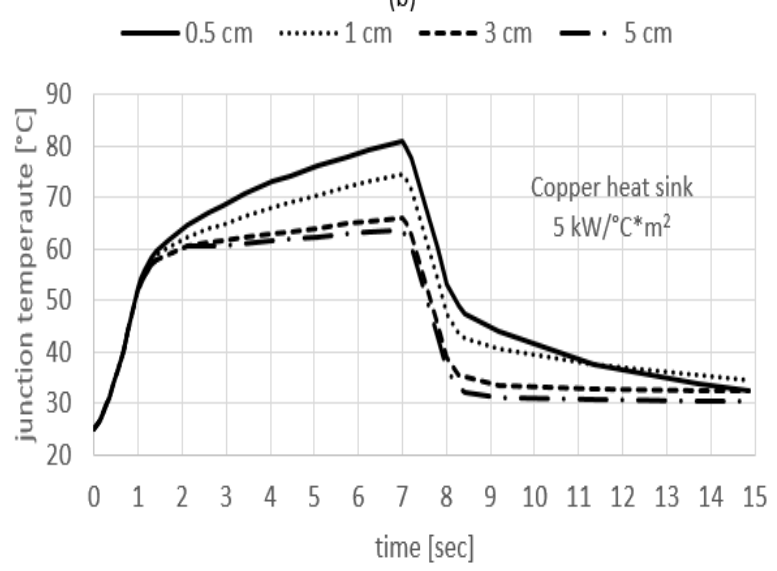

Fig. 4: (a) Junction temperature response for different thickness values of aluminum heat sink, (b) Junction temperature response for different thickness values of copper heat sink, both with a convection coefficient of $5 \mathrm{~kW} /{ }^{\circ} \mathrm{C}^{*} \mathrm{~m}^{2}$.

The Bode plot diagram of Fig. 5 illustrates the heat extraction ability for the different thickness values of the aluminum case by presenting the output heat to the ambient with respect to the input heat at the chip level. The $0.5 \mathrm{~cm}$ case demonstrates the highest ratio of output to input heat, due to its low thermal resistance. This heat extraction ability is not the selection criterion because it implies a low heat storage capability, a disadvantage for medium and long pulse applications. This comment is in accordance with Fig. 4a. The final choice is the aluminum $3 \mathrm{~cm}$ heat sink.

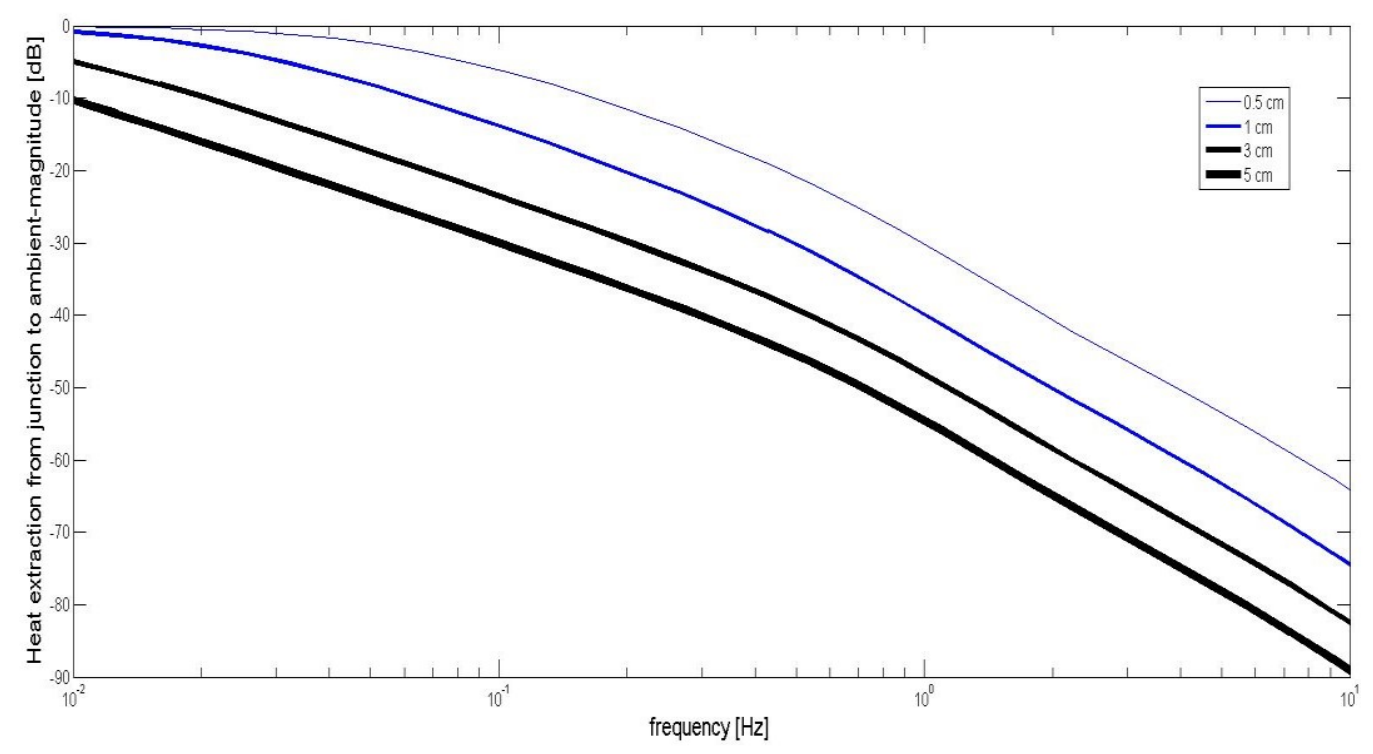

Fig. 5: Magnitude bode diagram for the heat extracted to the ambient with respect to the heat dissipated by the chip for the aluminum case

The next step is to test the aluminum heat sink with a highly effective water-cooling system, in order to identify a possible gain in terms of temperature swing reduction. Figure 6 shows the junction temperature response for the aluminum heat sink, if the convection coefficient increases to 10 $\mathrm{kW} /{ }^{\circ} \mathrm{C}^{*} \mathrm{~m}^{2}$. For the $0.5 \mathrm{~cm}$ the temperature swing and the maximum temperature are remarkably limited comparing to the $5 \mathrm{~kW} /{ }^{\circ} \mathrm{C}^{*} \mathrm{~m}^{2}$ case. For both the $3 \mathrm{~cm}$ and the $5 \mathrm{~cm}$ case, the enhanced convection does not offer a considerable advantage for the thermal stressing at chip level. Therefore, the selected cooling system is the one with a convection coefficient of $5 \mathrm{~kW} /{ }^{\circ} \mathrm{C}^{*} \mathrm{~m}^{2}$. 


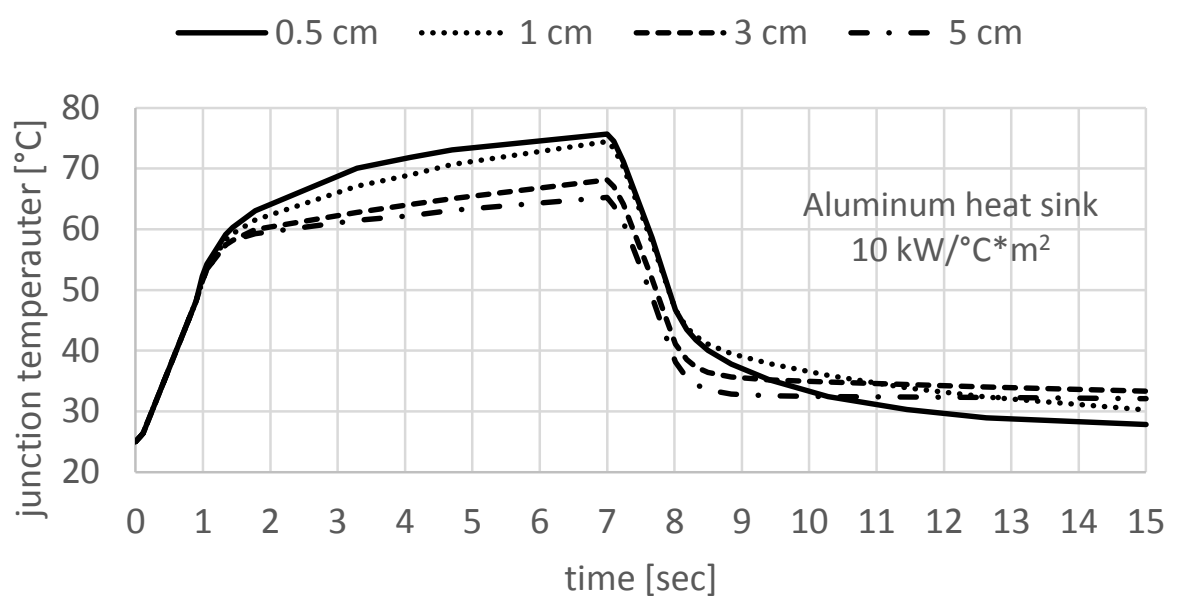

Fig. 6: Junction temperature response for different thickness values of aluminum heat sink and for an increased convection coefficient of $10 \mathrm{~kW} /{ }^{\circ} \mathrm{C}^{*} \mathrm{~m}^{2}$

Table III summarizes the temperature swing and the maximum temperature for the three cases; aluminum-5 kW/ $/{ }^{\circ} \mathrm{C}^{*} \mathrm{~m}^{2}$, copper-5 $\mathrm{kW} /{ }^{\circ} \mathrm{C}^{*} \mathrm{~m}^{2}$ and aluminum- $10 \mathrm{~kW} /{ }^{\circ} \mathrm{C}^{*} \mathrm{~m}^{2}$.

Table III: Junction temperature swing and maximum temperature for different materials, heat sink thickness and convection coefficient

\begin{tabular}{|c|c|c|c|}
\hline $\begin{array}{c}\text { Heat sink thickness } \\
{[\mathrm{cm}]}\end{array}$ & $\begin{array}{c}\text { For aluminum for } \\
5 \mathrm{~kW} /{ }^{\circ} \mathrm{C}^{*} \mathrm{~m}^{2} \\
\mathrm{~T}_{\text {swing }} / \mathrm{T}_{\max }\left[{ }^{\circ} \mathrm{C}\right]\end{array}$ & $\begin{array}{c}\text { For copper and } \\
5 \mathrm{~kW} /{ }^{\circ} \mathrm{C}^{*} \mathrm{~m}^{2} \\
\mathrm{~T}_{\text {swing }} / \mathrm{T}_{\max }\left[{ }^{\circ} \mathrm{C}\right]\end{array}$ & $\begin{array}{c}\text { For aluminum and } \\
10 \mathrm{~kW} /{ }^{\circ} \mathrm{C}^{*} \mathrm{~m}^{2} \text { in } \\
\mathrm{T}_{\text {swing }} / \mathrm{T}_{\max }\left[{ }^{\circ} \mathrm{C}\right]\end{array}$ \\
\hline 0.5 & $59.4 / 85$ & $55.8 / 80.84$ & $50.6 / 75.68$ \\
\hline 1 & $53.5 / 78$ & $49.5 / 74.55$ & $49.4 / 74.42$ \\
\hline 3 & $43.5 / 68.12$ & $41.1 / 66.12$ & $43.1 / 68.12$ \\
\hline 5 & $40.1 / 65$ & $38.72 / 63.72$ & $40.1 / 65.23$ \\
\hline
\end{tabular}

\section{Discussion on possible failures in the module structure - Lifetime estimation}

The aim of the investigated designs was to investigate the resulting thermal stressing of the power module and determine a lifetime according to the operating conditions. A lifetime estimation is needed based on the thermal stressing data that was obtained from the simulations. Reference [14] provides an estimated number of cycles to failure for the $10 \%$ of the number of samples. The critical parameters are the junction temperature swing, the junction maximum temperature and the cycle time. An alternative lifetime estimation in [14] uses the case minimum temperature and temperature swing as critical parameters. Although the conducted lifetime tests have a totally different profile than this application, a rough conclusion can be reached for the modules lifetime and probable defections. Based on the simulation results, a comparison is needed between the two estimations, in order to find the worst-case scenario that is the most pessimistic expected lifetime.

If the junction-based estimation is used, two different estimations are presented for bond-wire lift off and for chip solder delamination. The long pulse application could make the chip solder the dominant failure source. In Fig. 4, it is observed that the junction temperature is already close to the ambient temperature at the $15^{\text {th }}$ second of the cycle. Therefore, from [14] the cycle that is closer to this application has a duration of 10 seconds, temperature swing of $40^{\circ} \mathrm{C}$ and maximum temperature of $100{ }^{\circ} \mathrm{C}$. The module is expected to have a lifetime of at least $328^{*} 10^{6}$ cycles. A physical test of the modules with the specific load would be more accurate for the lifetime determination than this estimation.

In the same way as for the $3 \mathrm{~cm}$ case, as it can be seen in Table IV, the lifetime is calculated for the two materials, the different convection coefficients and the different thickness values. Again, the fact that is a long pulse application is taken into account for the lifetime estimation. The solder joint is considered 
responsible for the module future failure. In [14], the pulse length that is closer to the application of interest is 10 seconds. The maximum temperature for all cases is lower than in [14] that means that the lifetime estimation approach is conservative. Table IV shows the tendency in terms of lifetime for all the cases. It is observed that for the two thickest heat sinks, roughly no lifetime difference is expected, if the three cases are compared.

\section{Table IV: Lifetime estimation in terms of cycles for different materials, heat sink} thickness and convection coefficient

\begin{tabular}{|c|c|c|c|}
\hline $\begin{array}{c}\text { Heat sink thickness } \\
{[\mathrm{cm}]}\end{array}$ & $\begin{array}{c}\text { For aluminum for } \\
5 \mathrm{~kW} /{ }^{\circ} \mathrm{C}^{*} \mathrm{~m}^{2} \\
{[\text { million cycles] }}\end{array}$ & $\begin{array}{c}\text { For copper and } \\
5 \mathrm{~kW} /{ }^{\circ} \mathrm{C}^{*} \mathrm{~m}^{2} \\
{[\text { million cycles }]}\end{array}$ & $\begin{array}{c}\text { For aluminum and } \\
10 \mathrm{~kW} /{ }^{\circ} \mathrm{C}^{*} \mathrm{~m}^{2} \\
{[\text { million cycles] }}\end{array}$ \\
\hline 0.5 & 6.620 & 6.620 & 31.400 \\
\hline 1 & 31.400 & 31.400 & 31.400 \\
\hline 3 & 328 & 328 & 328 \\
\hline 5 & 328 & 328 & 328 \\
\hline
\end{tabular}

According to the estimation based on the case temperature measurements for solder joints failures of the conductor leads or substrate, the simulation results of a $13{ }^{\circ} \mathrm{C}$ temperature swing and a $38{ }^{\circ} \mathrm{C}$ maximum temperature show that no failure is expected from these parts. The case-based estimation could be applied more easily than the junction-based one, because the case is accessible for measurements.

\section{Conclusion}

In this paper, the heat sink composition, geometry and heat extraction means are investigated and determined in relation to the load cycles employed, in order to study the impact of on the temperature swings and the resulting lifetime. From the comparison of aluminum and copper as heat sink materials, it is observed that aluminum is as suitable as copper in this design. In spite of the fact that copper exhibits a better thermal performance than aluminum for most of the thermal parameters, the decrease of the maximum junction temperature and temperature swing, if a copper heat sink is used, is minor. The high specific thermal capacity of the aluminum compensates for the rest of its thermal properties for this application. The restriction that is set at the beginning for the heat sink surface area led to the increase of the heat sink thickness. The results are improved, if the heat sink thickness is increased, but the increase from $3 \mathrm{~cm}$ to $5 \mathrm{~cm}$ did not result in a significant advantage. The linear relation between the thermal resistance and the thickness, see (2), limits the thermal performance, after a thickness level. The thermal resistance prevents the heat extraction.

Furthermore, for mixed pulse applications, a thick heat sink may result in a slow cooling time, due to the high thermal resistance of the heat sink, enhancing the average and maximum junction temperature significantly. On the other hand, if the aim is the temperature swing reduction, a compromise is needed in the design for the absolute temperatures. A highly effective water cooling system would almost not change at all the thermal stressing for any of the $3 \mathrm{~cm}$ and $5 \mathrm{~cm}$ heat sinks. Two heat transfer coefficients were investigated and it was found that the heat sink thickness compensates for the convection coefficient. The convection coefficient influences essentially the system with the smallest heat sink of $0.5 \mathrm{~cm}$. The findings or the methodology could be extended to a module with an increased baseplate thickness cooled directly at this layer, even for customized solutions, where the comparison could be between copper and AlSiC, due to their lower thermal expansion coefficient comparing to aluminum for an improved lifetime.

\section{References}

[1] R. Bayerer, T. Herrmann, T. Licht, J. Lutz, and M. Feller, "Model for power cycling lifetime of IGBT modules - various factors influencing lifetime", $5^{\text {th }}$ International Conference on Integrated Power Systems (CIPS), 2008. 
[2] M. Ciappa, "Some reliability aspects of IGBT modules for high-power applications", ETH, 2000.

[3] J. Schulz-Harder, "Review on highly integrated solutions for power electronic devices", $5^{\text {th }}$ international conference on integrated power systems (CIPS), 2008.

[4] J. W. Kolar, U. Drofenik, J. Biela, M. L. Heldwein, H. Ertl*, T. Friedli and S. D. Round, "PWM Converter Power Density Barriers”, Power Conversion Conference, PCC '07, Nagoya, 2007.

[5] H. Huang and P.A. Mawby, "A Lifetime Estimation Technique for Voltage Source Inverters", IEEE TRANSACTIONS ON POWER ELECTRONICS, VOL. 28, NO. 8, AUGUST 2013.

[6] 5SNA 1600N170100 ABB datasheet.

[7] "Thermal equivalent circuit models", Application Note, V1.0, 2008, Infineon

[8] F. Incropera, "Principles of heat transfer", Wiley, 2013

[9] C. Yun, P. Malberti, M. Ciappa, and W. Fichtner," "Thermal Component Model for Electrothermal Analysis of IGBT Module Systems", IEEE TRANSACTIONS ON ADVANCED PACKAGING, VOL. 24, NO. 3, AUGUST 2001

[10] http://www.infomine.com/

[11] Jonas Ottoson, Thermal Modelling of Power Modules in a Hybrid Vehicle Application, Lund. University, 2013.

[12] Analysis of IGBT Module SystemsX. Wang, A. Castellazzi and P. Zanchetta, "Temperature Control for Reduced Thermal Cycling of Power Devices", 15th European Conference on Power Electronics and Applications (EPE), 2013

[13] L. Meysenc, M. Jylhäkallio and P. Barbosa, "Power Electronics Cooling Effectiveness Versus Thermal Inertia”, IEEE TRANSACTIONS ON POWER ELECTRONICS, VOL. 20, NO. 3, MAY 2005.

[14] Application note 5SYA 2043-04, ABB. 